\title{
Optimal storage properties of neural network models
}

\author{
E Gardner $†$ and B Derrida $\ddagger$ \\ † Department of Physics, Edinburgh University, Mayfield Road, Edinburgh, EH9 3JZ, UK \\ $\ddagger$ Service de Physique Theorique, CEN Saclay, F 91191 Gif sur Yvette, France
}

Received 29 May 1987

\begin{abstract}
We calculate the number, $p=\alpha N$ of random $N$-bit patterns that an optimal neural network can store allowing a given fraction $f$ of bit errors and with the condition that each right bit is stabilised by a local field at least equal to a parameter $K$. For each value of $\alpha$ and $K$, there is a minimum fraction $f_{\text {min }}$ of wrong bits. We find a critical line, $\alpha_{\mathrm{c}}(K)$ with $\alpha_{\mathrm{c}}(0)=2$. The minimum fraction of wrong bits vanishes for $\alpha<\alpha_{\mathrm{c}}(K)$ and increases from zero for $\alpha>\alpha_{\mathrm{c}}(K)$. The calculations are done using a saddle-point method and the order parameters at the saddle point are assumed to be replica symmetric. This solution is locally stable in a finite region of the $K, \alpha$ plane including the line, $\alpha_{c}(K)$ but there is a line above which the solution becomes unstable and replica symmetry must be broken.
\end{abstract}

\section{Introduction}

It is well known in the theory of spin glasses that when one considers an Ising Hamiltonian,

$$
\mathscr{H}=-\sum_{i, j} J_{i j} S_{l} S_{j}
$$

with random interactions $J_{i j}$, that there are a lot of metastable states (Bray and Moore 1980, 1981, De Dominicis et al 1980, Ettelaie and Moore 1985, Derrida and Gardner 1986, Gardner 1986) (at least at zero temperature). By definition, a metastable state at zero temperature is a spin configuration $\left\{S_{t}\right\}$ such that,

$$
S_{t}=\operatorname{sgn}\left(\sum_{j} J_{i j} S_{j}\right) .
$$

For randomly chosen interactions, $J_{i j}$, one can then try to calculate the number of these metastable states, the distribution of their energies, magnetisations, overlaps....

In the present paper, we address a different question; given $p$ spin configurations (which we call patterns), $\left\{S_{i}^{\mu}\right\}$ for $1 \leqslant i \leqslant N$ and $1 \leqslant \mu \leqslant p$, what is the probability that randomly chosen interactions $J_{i,}$ have these patterns as metastable states? This probability is related in a simple way to the volume in the space of all $J_{i \prime}$ in which equation (2) is satisfied for all sites $i$ and all patterns $\mu$. This question is an important one in the theory of neural networks where one usually represents the patterns one wants to store by spin configurations $\left\{S_{i}^{\mu}\right\}$ and the synapses by pair interactions, $J_{y}$.

Recent work has dealt with cases where is a simple storage rule giving each $J_{i j}$ as a function of the patterns $\left\{S_{i}^{\mu}\right\}$. For example, $J_{i j}=N^{-1} \Sigma_{\mu} S_{i}^{\mu} S_{1}^{\mu}$ (Hebb rule) (Hebb 1949, Little 1974, Hopfield 1982, Amit et al 1985a, b, 1987a, b), $J_{i j}=\operatorname{sgn}\left(N^{-1} \Sigma_{\mu} S_{\imath}^{\mu} S_{j}^{\mu}\right)$ 
or more complicated rules (Kohonen 1984, Personnaz et al 1985, Kanter and Sompolinsky 1987, Toulouse et al 1986, Parisi 1986, Mézard et al 1986). In general, when the number, $p=\alpha N$, of patterns is increased, there is a critical value $\alpha_{c}$ above which the system cannot store the patterns. $\alpha_{\mathrm{c}}$ depends on the rule used to calculate the $J_{i j}$.

The method we use here is similar to a maximum entropy approach since we consider that nothing is known about the $J_{i j}$ except that they try to make the patterns as metastable as possible. We will now define the problem more precisely. Consider $p$ patterns, $S_{i}^{\mu}= \pm 1(1 \leqslant i \leqslant N$ and $1 \leqslant \mu \leqslant p)$. These patterns are chosen at random and remain fixed. For each choice of the $J_{i j}$, we call $p_{w}\left(\left\{J_{i j}\right\}\right)$ the number of patterns, $\mu$, such that a given site $i$ is wrong,

$$
p_{k} \cdot\left(\left\{J_{i j}\right\}\right)=\sum_{\mu=1}^{p}\left[1-\theta\left(S_{i}^{\mu} \sum_{j} J_{i j} S_{j}^{\mu}\left(\sum_{j} J_{i j}^{2}\right)^{-1 / 2}-K\right)\right] .
$$

We say here that a site $i$ is right for pattern $\mu$ if $S_{i}^{\mu}$ is not only parallel to the local field, $h_{i}^{\mu}=\Sigma_{j} J_{i j} S_{j}^{\mu} /\left(\Sigma_{j} J_{i j}^{2}\right)^{1 / 2}$ but if also $h_{i}^{\mu} S_{i}^{\mu}$ is greater than a minimal value $K$. Of course, a wrong site is by definition a site which is not right. For a given number $p$ of random patterns, one can ask what is the volume in the space of the $J_{i j}$ which gives a certain fraction $f$ of wrong patterns at site $i$. If one tries to minimise $f$ for a given $p$, then one has to solve an optimisation problem whose cost function is given by (3). It is clear that if a choice of $J_{i j}$ gives a certain $f$, the choice $\lambda J_{i j}$ gives exactly the same $f$. So one needs to choose a constraint on the $J_{i j}$ in order to have a finite volume. In the present paper, two constraints will be considered, a spherical one in $\$ \S 2$ and 3 ,

$$
\sum_{j} J_{i j}^{2}=N
$$

and an Ising one,

$$
J_{i j}= \pm 1
$$

in $\S 4$. The basic quantity that we will consider is a partition function $Z(h)$ defined by

$$
Z(h)=\left\langle\exp \left[-h p_{w}\left(\left\{J_{i j}\right\}\right)\right]\right\rangle_{\left\{J_{i j}\right\}}
$$

where $p_{w}$ is given by (3). The $J_{i}$, here play the role of the dynamical variables (which are in 'thermal equilibrium') whereas the $S_{i}^{\mu}$ are the quenched variables. Expression (6) can be easily transformed into

$$
Z(h)=\left\langle\prod_{\mu=1}^{p}\left[\mathrm{e}^{-h}+\left(1-\mathrm{e}^{-h}\right) \theta\left(S_{i}^{\mu} \sum_{j} J_{i j} S_{j}^{\mu} N^{-1 / 2}-K\right)\right]\right\rangle_{\left\{J_{i j}\right\}} .
$$

Several quantities of interest can be computed from a knowledge of $Z(h)$. For example, the average, $\left\langle p_{w}\right\rangle=p f$, the number of wrong patterns at site $i$ is given by

$$
p f=\left\langle p_{w}\right\rangle=-\frac{\mathrm{d}}{\mathrm{d} h} \ln Z(h) .
$$

In the limit, $h \rightarrow \infty$, the minimal fraction $f_{\min }$ of wrong patterns at site $i$ (i.e. the fraction $f$ obtained for the optimal choice of the $J_{i j}$ ) is given by

$$
p f_{\min }=\lim _{h \rightarrow x}-\frac{d}{d h} \ln Z(h) .
$$


It is clear from the above expression that the meaningful quantity to study is $\ln Z(h)$. We have here restricted consideration to the single-site problem. However, since all quantities we are interested in are derived from derivatives of $\ln Z(h)$, this problem is equivalent to the complete problem where the constraints (3) are fixed at all sites provided that the variables $J_{i j}$ at different sites $i$ are independent. This need not of course be true if, for example, a symmetry constraint $J_{i j}=J_{j i}$ is imposed.

In $\S 2$, we calculate $\overline{\ln Z}$, the typical value of $\ln Z$, using the replica method (the bar means an average on the patterns) for the spherical constraint (4). From this expression of $\ln Z(h)$, we obtain $f_{\min }$ as a function of $\alpha=p / N$ and $K$. We also give the expression $\alpha_{\mathrm{c}}(K)$ such that $f_{\min }=0$ for $\alpha<\alpha_{\mathrm{c}}$. In $\S 3$, the stability of the replica symmetric solution of $\S 2$ is analysed. The solution is always stable when $f=0$ (provided $K$ is positive).

This seems reasonable because the space of solutions $J_{i j}$ is connected; any two solutions can be continuously deformed into one another (i.e. there is a single valley). If, however, the mean fraction of wrong bits is positive, different solutions can correspond to errors in different bits and the solution space need not be connected (the set of solutions may be composed of many valleys). We find that the solution is stable in a finite region of the space, $K, \alpha, f$. On the surface of this space where $f$ is a minimum, the replica-symmetric solution is stable if $\alpha$ is small enough and there is a line above which it becomes unstable. This therefore provides a new example of a problem where replica symmetry has to be broken. In $\$ 4$, we will discuss the Ising constraint (5). Since the number of possible states of the $J_{i j}$ is finite, the entropy can be calculated. We show that, in the replica symmetric approximation, the entropy or logarithm of the number of solutions at the minimum value of $f$ is always $-\infty$ and the replicasymmetric solution is unstable. The calculation of the number of solutions is only valid in a finite region of the space $K, f, \alpha$ but not at values of $\alpha$ and $K$ which give $f_{\min }(\geqslant 0)$.

\section{The spherical model}

In order to average $\ln Z(h)$, we use the replica method

$$
\overline{\ln Z(h)}=\lim _{n \rightarrow 0} \frac{\overline{Z^{n}(h)}-1}{n} .
$$

When one introduces replicas $(1 \leqslant \alpha \leqslant n)$, each variable $J_{i j}$ is replicated, $J_{i j}^{\alpha}$, and with the spherical constraint $(4), \overline{Z^{n}(h)}$ is given by

$$
\begin{aligned}
\overline{Z^{n}(h)}=C \int & \prod_{\alpha=1}^{n} \frac{\mathrm{d} \varepsilon_{\alpha}}{2 \pi} \int \prod_{j} \mathrm{~d} J_{i j}^{\alpha} \exp \mathrm{i} \varepsilon_{\alpha}\left(\sum_{j}\left(J_{i j}^{\alpha}\right)^{2}-N\right) \\
& \times \prod_{\mu}\left[\mathrm{e}^{-h}+\left(1-\mathrm{e}^{-\mathrm{h}}\right) \theta\left(N^{-1 / 2} S_{i}^{\mu} \sum_{j} J_{i j} S_{j}^{\mu}-K\right)\right]
\end{aligned}
$$

where the constant $C$ is just the normalisation of the volume of the space of $J_{i j}$,

$$
C=\left[\frac{1}{2 \pi} \int \mathrm{d} \varepsilon \int \prod_{j} \mathrm{~d} J_{i j} \exp \mathrm{i} \varepsilon\left(\sum_{j} J_{i j}^{2}-N\right)\right]^{-n} .
$$

In the thermodynamic limit $(N \rightarrow \infty), \overline{Z^{n}(h)}$ can be calculated using a saddle-point 
method since it can be written

$$
\begin{aligned}
\overline{Z^{n}(h)}=\int \sum_{\alpha<\beta} & \mathrm{d} q_{\alpha \beta} \frac{\mathrm{d} \varphi_{\alpha \beta}}{(2 \pi / N)} \prod_{\alpha} \frac{\mathrm{d} \varepsilon_{\alpha}}{2 \pi} \exp N \\
& \times\left(\frac{p}{N} G_{0}\left(\left\{q_{\alpha \beta}\right\}\right)+G_{1}\left(\left\{\varphi_{\alpha \beta}\right\},\left\{\varepsilon_{\alpha}\right\}\right)+i \sum_{\alpha<\beta} \varphi_{\alpha \beta} q_{\alpha \beta}\right) .
\end{aligned}
$$

The derivation of (13) and the expressions for $G_{0}\left(\left\{q_{\alpha \beta}\right\}\right)$ and $G_{1}\left(\left\{\varphi_{\alpha \beta}\right\},\left\{\varepsilon_{\alpha}\right\}\right)$ are given in appendix 1 . If one assumes that $\ln Z(h)$ is given by a replica-symmetric saddle point in the limit $n \rightarrow 0$,

$$
q_{\alpha \beta}=q \quad \varphi_{\alpha \beta}=\mathrm{i} F \quad \varepsilon_{\alpha}=\mathrm{i} E
$$

then one gets (see (A1.17) and (A1.18)),

$$
\frac{\ln Z(h)}{N}=\operatorname{extr}_{E, F, q} \frac{1}{n}\left[\alpha G_{0}(q)+G_{1}(\mathrm{i} F, \mathrm{i} E)+\frac{1}{2} n F q\right]
$$

where $\alpha=p / N$ and

$$
\begin{aligned}
G_{0}(q)=n \int_{-\infty}^{\infty} & \frac{\exp \left(-t^{2} / 2\right)}{(2 \pi)^{1 / 2}} \mathrm{~d} t \\
& \times \ln \left(1+\left(e^{-h}-1\right) \int_{-\infty}^{\left(K-t q^{1 / 2}\right)(1-q)^{-1 / 2}}(2 \pi)^{-1 / 2} \exp \left(-\lambda^{2} / 2\right) \mathrm{d} \lambda\right) \\
& G_{1}(\mathrm{i} F, \mathrm{i} E)=n\left[E+\frac{1}{2} \ln 2 \pi-\frac{1}{2} \ln (2 E+F)+\frac{1}{2} F /(2 E+F)\right] .
\end{aligned}
$$

This leads to the saddle-point equations

$$
\begin{aligned}
& 1=\frac{2(E+F)}{(2 E+F)^{2}} \\
& q=\frac{F}{(2 E+F)^{2}}
\end{aligned}
$$

and

$$
F+\alpha \frac{\mathrm{d}}{\mathrm{d} q}\left(\frac{1}{n} G_{0}(q)\right)=0 .
$$

From (18) and (19) it is easy to calculate $E$ and $F$ as functions of $q$, and one gets

$$
F=\frac{q}{(1-q)^{2}} \quad E=\frac{1-2 q}{2(1-q)^{2}}
$$

and therefore the replica-symmetric solution leads to

$$
\begin{aligned}
\frac{\overline{\ln Z(h)}}{N}=\operatorname{extr}_{q}\left[\alpha \int_{-x}^{\infty} \frac{\exp \left(-t^{2} / 2\right)}{(2 \pi)^{1 / 2}} \mathrm{dt}\right. \\
\quad \times \ln \left(1+\left(\mathrm{e}^{-h}-1\right) \int_{-x}^{\left(K-\left(q^{1 / 2}\right)(1-q)^{-1 / 2}\right.}(2 \pi)^{1 / 2} \exp \left(-\lambda^{2} / 2\right) \mathrm{d} \lambda\right) \\
\left.+\frac{1}{2} \ln 2 \pi+\frac{1}{2} \ln (1-q)+\frac{1}{2}(1-q)^{-1}\right] .
\end{aligned}
$$


From this expression, one can deduce the minimal fraction $f_{\min }$ of wrong spins: in the limit $h \rightarrow \infty$ and $q \rightarrow 1$, one gets for the leading term

$$
\begin{aligned}
\frac{\overline{\ln Z(h)}}{N}=\operatorname{extr}_{q} & {\left[\alpha \left(-\int_{K-[2 h(1-q)]^{1 / 2}}^{K} \exp \left(-t^{2} / 2\right) \frac{\mathrm{d} t}{(2 \pi)^{1 / 2}} \frac{(t-K)^{2}}{2(1-q)}\right.\right.} \\
& \left.\left.-h \int_{-\infty}^{K-[2 h(1-q)]^{1 / 2}} \frac{\exp \left(-t^{2} / 2\right)}{(2 \pi)^{1 / 2}} \mathrm{~d} t\right)+\frac{1}{2}(1-q)^{-1}\right]
\end{aligned}
$$

which can be rewritten as

$$
\begin{gathered}
\frac{\overline{\ln Z(h)}}{N}=-h \alpha f_{\min }=h \operatorname{extr}_{x}\left(-\alpha \int_{K-x}^{K} \frac{\mathrm{d} t \exp \left(-t^{2} / 2\right)}{(2 \pi)^{1 / 2}} \frac{(t-K)^{2}}{x^{2}}\right. \\
\left.-\alpha \int_{-\infty}^{\kappa-x} \frac{\mathrm{d} t \exp \left(-t^{2} / 2\right)}{(2 \pi)^{1 / 2}}+\frac{1}{x^{2}}\right) .
\end{gathered}
$$

Setting the derivative of (24) with respect to $x$ to zero, one finds that there is an optimal $x$ only if $\alpha>\alpha_{\mathrm{c}}$ where

$$
\alpha_{\mathrm{c}}=\left(\int_{-x}^{K} \frac{\mathrm{d} t}{(2 \pi)^{1 / 2}} \exp \left(-t^{2} / 2\right)(t-K)^{2}\right)^{-1} .
$$

The function $\alpha_{c}(K)$ is plotted in figure 1. $\alpha_{c}(0)$ is equal to 2 in agreement with known results (Cover 1965, Venkatesh 1986a, b).

If $\alpha<\alpha_{\mathrm{c}}$, the extremum is given by

$$
x=\infty
$$

and therefore

$$
f_{\min }=0
$$

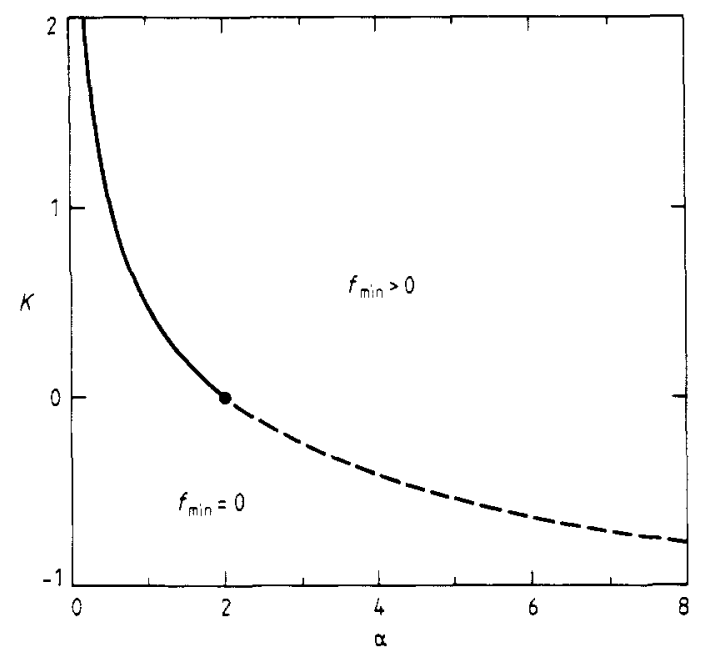

Figure 1. The critical line $\alpha_{c}(K)$. For $\alpha<\alpha_{c}(K)$, the minimum fraction of wrong bits $f_{\min }=0$. The line is calculated assuming replica symmetry. For $K>0$ (full curve) the replica-symmetric saddle point is stable around the line $\alpha_{c}(K)$ whereas for $K<0$ (broken curve) it is unstable. 
whereas for $\alpha>\alpha_{\mathrm{c}}, x$ is a solution of

$$
\alpha \int_{K-x}^{K} \frac{\mathrm{d} t}{(2 \pi)^{1 / 2}} \exp \left(-t^{2} / 2\right)(t-K)^{2}=1
$$

and $f_{\min }$ given by

$$
f_{\min }=\int_{-x}^{K-x} \frac{\mathrm{d} t}{(2 \pi)^{1 / 2}} \exp \left(-t^{2} / 2\right)
$$

$f_{\min }$ is plotted in figure 2 as a function of $\alpha$ for $K=0,0.5$ and 1.

These expressions of $\alpha_{c}(K)$ and $f_{\min }$ have been obtained assuming replica symmetry. We will see in the next section that the replica-symmetric solution becomes unstable when $\alpha$ increases. Nevertheless for $K>0$, the whole line $\alpha_{c}(K)$ lies in a region where the replica-symmetric solution is stable. Recent numerical results (Krauth and Mézard 1987) seem to agree with expression (25).

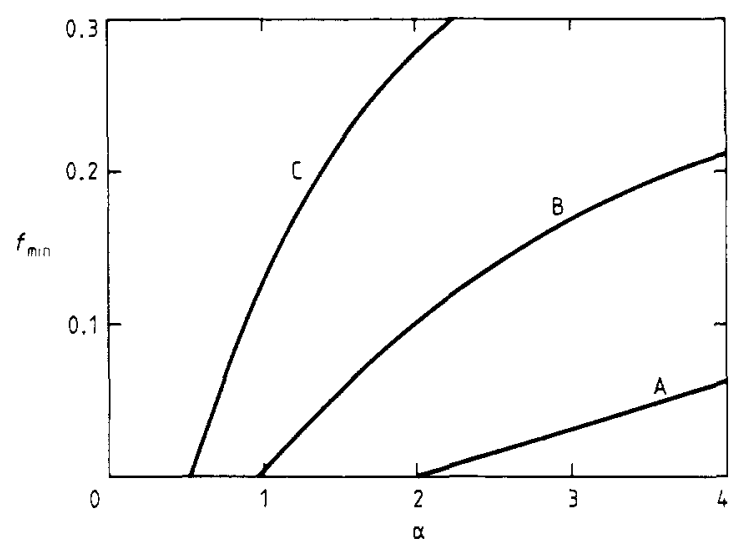

Figure 2. The minimum fraction, $f_{\min }$, of wrong bits as a function of $\alpha$ for $K=0$ (curve A), $0.5(\mathrm{~B})$ and $1.0(\mathrm{C})$.

\section{Stability of the replica symmetric solution}

An instability of the replica-symmetric solution (14) to the mean-field equations derived from equation (13) is determined by a sign change in (at least) one of the eigenvalues of quadratic fluctuations in the order parameters $q_{\alpha \beta}, \Phi_{\alpha \beta}$ and $\varepsilon_{\alpha}$ around the saddle point.

We will first consider the problem of diagonalising the matrices of second derivatives with respect to $q_{\alpha \beta}$ of $G_{0}$ and of second derivatives with respect to i $\Phi_{\alpha \beta}$ and $\mathrm{i} \varepsilon_{\alpha}$ of $G_{1}$ separately. At the replica-symmetric saddle point, these matrices have the same symmetry properties as the matrix of quadratic fluctuations in the Edwards-Anderson (1975) order parameters in the Sherrington-Kirkpatrick (1975) model around the replica-symmetric solution. The eigenvectors (de Almeida and Thouless 1978) can be divided into two types: those which have the same eigenvalues as longitudinal fluctuations in the replica-symmetric space and those which are transverse to that space and have eigenvalues different from the longitudinal ones. 
We will consider first the transverse eigenvectors and eigenvalues which are distinct from the longitudinal ones. The transverse eigenvectors of $G_{0}$ in terms of fluctuations in the variables $q_{\alpha \beta}$ are parallel to those of $G_{1}$ in terms of the variables $i \Phi_{\alpha \beta}$ and have no component in the direction of fluctuations in the variables $i \varepsilon_{\alpha}$. In each case there is a unique $\frac{1}{2} n(n-3)$-fold degenerate eigenvalue $\gamma_{1}$ and $\gamma_{2}$ respectively. These eigenvectors and eigenvalues are calculated in appendix 2 .

The analysis of the longitudinal fluctuations could also be done. It turns out that there are three distinct eigenvalues in the longitudinal direction, and also a set of $3(n-1)$ transverse eigenvalues which are degenerate with the longitudinal ones. Since the replica-symmetric solution for $E, F$ and $q$ is unique, the solution should be stable with respect to fluctuations in this three-dimensional space, and therefore these eigenvectors should not lead to an instability.

The transverse eigenvalues of the quadratic fluctuations in the function

$$
\alpha G_{0}\left(q_{\alpha \beta}\right)+G_{1}\left(\varepsilon_{\alpha}, \varphi_{\alpha \beta}\right)+\mathrm{i} q_{\alpha \beta} \varphi_{\alpha \beta}
$$

on the right-hand side of equation (13) are therefore given by the two eigenvalues of the matrix,

$$
\left(\begin{array}{cc}
\alpha \gamma_{1} & 1 \\
1 & \gamma_{2}
\end{array}\right)
$$

In the limit $\alpha \rightarrow 0$, and hence $q \rightarrow 0$, the product of these eigenvalues is -1 . The replica-symmetric result is correct in this limit since it is simply an integral over the phase space of couplings $J_{i j}^{\alpha}$, and the result therefore must be stable. The sign is negative because of the change of variable from $\Phi_{\alpha \beta}$ to i $\Phi_{\alpha \beta}$. The product can also be evaluated in the limit $\alpha \rightarrow \alpha_{c}, q \rightarrow 1$. A sign change in the product implies that one of the eigenvalues has changed sign for some value of $\alpha$ less than $\alpha_{c}$. The replicasymmetric solution is unstable and the cost function calculations of the previous section therefore invalid if

$$
\alpha_{c} \gamma_{1}, \gamma_{2}>1
$$

From appendix 2, (31) holds if

$$
x \frac{1}{(2 \pi)^{1 / 2}} \exp \left[(K-x)^{2} / 2\right]>K \int_{K-x}^{K} \frac{\mathrm{d} t \exp \left(-t^{2} / 2\right)}{(2 \pi)^{1 / 2}}(K-t) .
$$

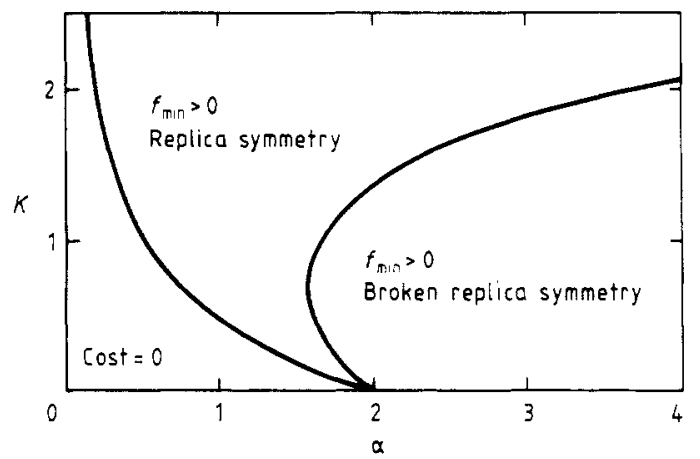

Figure 3. The critical line $\alpha_{c}(K)$ and the line where the replica-symmetric solution becomes unstable. 
In the limit $x \rightarrow \infty$ or the fraction of errors $\rightarrow 0$, the product of eigenvectors has the same sign as at $\alpha=0$. However, as the fraction of errors increases, $x$ decreases and the solution becomes unstable. For example, for small $x(x \ll K)$ inequality (32) is always satisfied and the solution is always unstable. The instability line in the $\alpha, K$ plane is plotted in figure 3 .

\section{The Ising model}

In this section, the calculation of $\S 2$ will be repeated for the Ising constraint (equation (5)). Since the number of points in the phase space of interactions is finite for this constraint (for finite $N$ ), the total number $\Omega$ of solutions for a fraction $f$ of wrong bits is given by

$$
\overline{\ln \Omega}=\overline{\ln Z(h)}-h \frac{\overline{\mathrm{d} \ln Z(h)}}{\mathrm{d} h}
$$

where $h$ can be calculated as a function of $f$ using equation (8). In the replica approach, we have again to consider $\overline{Z^{n}(h)}$,

$\overline{Z^{n}(h)}=\int \prod_{\alpha<\beta} \mathrm{d} q_{\alpha \beta} \frac{\mathrm{d} \varphi_{\alpha \beta}}{2 \pi} \exp N\left(\alpha G_{0}\left(\left\{q_{\alpha \beta}\right\}\right)+G_{1}\left(\left\{\varphi_{\alpha \beta}\right\}\right)+\mathrm{i} \sum_{\alpha<\beta} \varphi_{\alpha \beta} q_{\alpha \beta}\right)$

where $G_{0}$ is given by equation (16) while $G_{1}$ is given assuming replica symmetry (14) by

$$
G_{1}=n\left(\int_{-x}^{\infty} \frac{\mathrm{d} u \exp \left(-u^{2} / 2\right)}{(2 \pi)^{1 / 2}}\left\{\ln [2 \cosh (u \sqrt{F})]-\frac{1}{2} F\right\}\right)
$$

(see A1.21). The mean-field equation for $F$ implies

$$
F=\frac{2}{\pi(1-q)^{2}}
$$

as $q \rightarrow 1$ and

$$
G_{1}+\frac{n F q}{2} \rightarrow \frac{n}{\pi(1-q)}
$$

The cost function, $f$, can therefore be expressed as an extremum over $x$ as in equation (24) except that the last $x^{-2}$ term is multiplied by a factor $2 / \pi$. This is equivalent to multiplying $\alpha$ by a factor of $\pi / 2$, and so equation (27) becomes

$$
\frac{\alpha_{\mathrm{c}} \pi}{2} \int_{K-x}^{K} \frac{\exp \left(-t^{2} / 2\right)}{(2 \pi)^{1 / 2}} \mathrm{~d} t(t-K)^{2}=1
$$

while $f_{\min }$ is given by equation (28). In particular, in the limit $f_{\min } \rightarrow 0, x \rightarrow \infty$ and $K=0$, the upper storage capacity $\alpha_{\mathrm{c}}$ is given by

$$
\alpha_{\mathrm{c}}=4 / \pi
$$

In contrast to the spherical model calculation where there is a region of the $\alpha, K$ plane where the replica-symmetric solution is stable, and $f_{\min }>0$, the Ising calculation is always incorrect as $q \rightarrow 1$. Clearly equation (39) is incorrect since the maximum amount 
of information which can be stored must be less than the number of bonds, and so $\alpha_{\mathrm{c}}$ must be less than 1. The entropy or logarithm of the number of solutions can be calculated and as $q \rightarrow 1$ or $f_{\min } \rightarrow 0$ we find

$$
\overline{\ln \Omega} / N \sim \ln (1-q) \rightarrow-\infty
$$

implying an infinite negative entropy. The stability calculation of $\S 3$ can also be repeated for this case and the product of the distinct transverse eigenvalues tends to infinity in this limit, implying that there is a sign change in one of the eigenvalues for some value of $q$ less than 1 .

The Ising calculation, of the number of solutions $\Omega$ of equation (33), is valid only for sufficiently small values of $q$. Specifically, there is a surface in the $\alpha, f, K$ space on one side of which replica symmetry must be broken. In particular, the calculation of $f_{\min }$ always requires replica-symmetry breaking.

\section{Conclusions}

In this paper, we have calculated the fractional volume of phase space with a given value of $K$ and of the fraction of wrong bits $f$. In particular the minimum cost function, $f_{\min }$, has been calculated. All of these results, however, have been obtained assuming replica symmetry for the order parameters at the saddle point. For both constraints-the spherical one (4) and the Ising one (5)-there is a surface in the space $K, \alpha, f$ on one side of which this replica-symmetric solution is unstable and the calculations must therefore be incorrect. For the spherical constraint, the calculations are stable in a finite region of the $K, f_{\min }$ plane around $f_{\min }=0$ and $K>0$, whereas in the Ising case, the solution is unstable throughout this plane.

The calculational method of $\S 2$ has been used in other situations, for example, to deal with correlated patterns (Gardner 1987, 1988a). It can also be extended in order to calculate the size of basins of attraction in a diluted version of this model (Gardner 1988 b) where finite values of $K>0$ do imply finite basins of attraction which increase with the magnitude of $K$.

Since explicit solutions for the optimal $J_{i j}$ do not exist, it is important to have numerical methods for constructing them. For $f=0$, extensions of the perceptron learning algorithm (Rosenblatt 1962, Minsky and Papert 1969) do exist which converge to solutions provided these solutions exist (Gardner 1988a, Krauth and Mézard 1987). These algorithms therefore generate exact storage with finite basins of attraction provided such solutions exist. Other algorithms which aim to give solutions with finite basins of attraction have also been discussed (Gardner et al 1987, Diederich and Opper 1987, Poppel and Krey 1987).

\section{Acknowledgments}

We would like to thank H Gutfreund, M Mézard and G Toulouse for useful discussions. EG thanks the Service de Physique Theorique for their hospitality whilst in Saclay. Financial support for EG was from a Dewar Fellowship from the University of Edinburgh. 


\section{Appendix 1}

In this appendix, we show how equation (11) leads to expressions (13) and (14) and we calculate the explicit expressions for the functions $G_{0}$ and $G_{1}$ for the replicasymmetric solution. If the $\theta$ functions in (11) are replaced by

$$
\begin{aligned}
& \theta\left(\frac{1}{\sqrt{N}} \sum_{j} S_{i}^{\mu} J_{i j}^{\alpha} S_{j}^{\mu}-K\right)=\frac{1}{2 \pi} \int_{K}^{\infty} \mathrm{d} \lambda_{\mu}^{\alpha} \\
& \times \int_{-\infty}^{\infty} \mathrm{d} x_{\mu}^{\alpha} \exp \left[i x_{\mu}^{\alpha}\left(\lambda_{\mu}^{\alpha}-\sum_{j} J_{i j}^{\alpha} N^{-1 / 2} S_{i}^{\mu} S_{j}^{\mu}\right)\right] .
\end{aligned}
$$

Then $\overline{Z^{n}(h)}$ becomes

$$
\begin{aligned}
\overline{Z^{n}(h)}=C \int & \prod_{\alpha} \frac{1}{2 \pi} \mathrm{d} \varepsilon_{\alpha} \int \prod_{j} \mathrm{~d} J_{i j}^{\alpha} \exp \left[\mathrm{i} \varepsilon_{\alpha}\left(\sum_{j}\left(J_{i j}^{\alpha}\right)^{2}-N\right)\right] \\
& \times \prod_{\mu}\left(\mathrm{e}^{-h} \int_{-\infty}^{K} \frac{\mathrm{d} \lambda_{\mu}^{\alpha}}{2 \pi}+\int_{K}^{\infty} \frac{\mathrm{d} \lambda_{\mu}^{\alpha}}{2 \pi}\right) \int_{-\infty}^{\infty} \mathrm{d} x_{\mu}^{\alpha} \\
& \times \exp \left[\mathrm{i} x_{\mu}^{\alpha}\left(\lambda_{\mu}^{\alpha}-\sum_{j} J_{i j}^{\alpha} N^{-1 / 2} S_{i}^{\mu} S_{J}^{\mu}\right)\right] .
\end{aligned}
$$

Averaging over the patterns, $S_{j}^{\mu}$, we get a coupling between replicas

$$
\exp \left(-\mathrm{i} \sum_{\alpha} x_{\mu}^{\alpha} N^{-1 / 2} J_{i j}^{\alpha} S_{i}^{\mu} S_{j}^{\mu}\right)=\cos \left(\sum_{\alpha} x_{\mu}^{\alpha} J_{i j}^{\alpha} N^{-1 / 2}\right) \text {. }
$$

Where $N$ is large, it is only necessary to keep the first term in the expansion of the cosine

$$
\ln \cos \left(\sum_{\alpha} x_{\mu}^{\alpha} J_{i j}^{\alpha} N^{-1 / 2}\right)=-\frac{1}{2 N}\left(\sum_{\alpha} x_{\mu}^{\alpha} J_{i j}^{\alpha}\right)^{2}
$$

and $\overline{Z^{n}(h)}$ therefore becomes

$$
\begin{aligned}
\overline{Z^{n}(h)}=C \int & \prod_{\alpha} \frac{\mathrm{d} \varepsilon_{\alpha}}{2 \pi} \int_{0} \mathrm{~d} J_{i j}^{\alpha} \prod_{\mu}\left(\mathrm{e}^{-h} \int_{-\infty}^{\kappa} \frac{\mathrm{d} \lambda_{\mu}^{\alpha}}{2 \pi}+\int_{K}^{\infty} \frac{\mathrm{d} \lambda_{\mu}^{\alpha}}{2 \pi}\right) \\
& \times \prod_{\mu} \int_{-\infty}^{\infty} \mathrm{d} x_{\mu}^{\alpha} \exp \left[\Phi\left(\left\{\varepsilon_{\alpha}\right\},\left\{J_{i j}^{\alpha}\right\},\left\{x_{\mu}^{\alpha}\right\},\left\{\lambda_{\mu}^{\alpha}\right\}\right)\right]
\end{aligned}
$$

where

$$
\Phi=\mathrm{i} \sum_{\alpha} \varepsilon_{\alpha}\left(\sum_{j}\left(J_{i j}^{\alpha}\right)^{2}-N\right)+\mathrm{i} \sum_{\alpha} \sum_{\mu} x_{\mu}^{\alpha} \lambda_{\mu}^{\alpha}-\frac{1}{2 N} \sum_{\mu} \sum_{j}\left(\sum_{\alpha} x_{\mu}^{\alpha} J_{i j}^{\alpha}\right)^{2}
$$

which can be easily transformed into

$$
\begin{gathered}
\Phi=\mathrm{i} \sum_{\alpha} \varepsilon_{\alpha}\left(\sum_{j}\left(J_{i j}^{\alpha}\right)^{2}-N\right)+\mathrm{i} \sum_{\alpha} \sum_{\mu} x_{\mu}^{\alpha} \lambda_{\mu}^{\alpha}-\frac{1}{2} \sum_{\mu} \sum_{\alpha}\left(x_{\mu}^{\alpha}\right)^{2} \\
-\sum_{\mu} \sum_{\alpha<\beta} x_{\mu}^{\alpha} x_{\mu}^{\beta}\left(\frac{1}{N} \sum_{j} J_{i j}^{\alpha} J_{i j}^{\beta}\right)
\end{gathered}
$$

where we have used the fact that $\Sigma_{j}\left(J_{i j}^{\alpha}\right)^{2}=N$. For each pair of replicas, one can then introduce

$$
1=\int \frac{\mathrm{d} q_{\alpha \beta} \mathrm{d} \varphi_{\alpha \beta}}{(2 \pi / N)} \exp \left[\mathrm{i} \varphi_{\alpha \beta}\left(N q_{\alpha \beta}-\sum_{j} J_{i j}^{\alpha} J_{i j}^{\beta}\right)\right]
$$


Then, one obtains

$$
\begin{aligned}
\overline{Z^{n}(h)}=\int \prod_{\alpha<\beta} & \frac{\mathrm{d} q_{\alpha \beta} \mathrm{d} \varphi_{\alpha \beta}}{2 \pi} \prod_{\alpha} \frac{\mathrm{d} \varepsilon_{\alpha}}{2 \pi} \\
& \quad \times \exp N\left(\alpha G_{0}\left(\left\{q_{\alpha \beta}\right\}\right)+\mathrm{i} \sum_{\alpha<\beta} \varphi_{\alpha \beta} q_{\alpha \beta}+G_{1}\left(\left\{\varphi_{\alpha \beta}\right\},\left\{\varepsilon_{\alpha}\right\}\right)\right)
\end{aligned}
$$

where

$$
\begin{aligned}
\exp G_{0}\left(\left\{q_{\alpha \beta}\right\}\right) & =\prod_{\alpha}\left(e^{-h} \int_{-\infty}^{\kappa} \frac{\mathrm{d} \lambda^{\alpha}}{2 \pi}+\int_{K}^{\infty} \frac{\mathrm{d} \lambda^{\alpha}}{2 \pi}\right) \\
& \times \int_{-\infty}^{\infty} \mathrm{d} x^{\alpha} \exp \left(\mathrm{i} x^{\alpha} \lambda^{\alpha}-\frac{1}{2}\left(x^{\alpha}\right)^{2}-\sum_{\alpha<\beta} x^{\alpha} x^{\beta} q_{\alpha \beta}\right)
\end{aligned}
$$

where $p$ is the number of patterns and

$$
\exp G_{1}\left(\left\{\varphi_{\alpha \beta}\right\},\left\{\varepsilon_{\alpha}\right\}\right)=\prod_{\alpha} \mathrm{d} J_{i}^{\alpha} \exp \left(\mathrm{i} \sum_{\alpha} \varepsilon_{\alpha}\left[\left(J_{i}^{\alpha}\right)^{2}-1\right]-\mathrm{i} \sum \varphi_{\alpha \beta} J_{i}^{\alpha} J_{i}^{\beta}\right) \text {. }
$$

Expressions (A1.10) and (A1.11) can be greatly simplified when the calculation is limited to the replica-symmetric subspace

$$
\begin{array}{ll}
q_{\alpha \beta}=q & \alpha \neq \beta \\
\varphi_{\alpha \beta}=\varphi=\mathrm{i} F & \alpha \neq \beta \\
\varepsilon_{\alpha}=\varepsilon=\mathrm{i} E & \text { for all } \alpha
\end{array}
$$

and one obtains

$$
\begin{aligned}
G_{0}(q)=\ln \left\{\int_{-\infty}^{\infty}\right. & \frac{\mathrm{e}^{-t^{2} / 2} \mathrm{~d} t}{(2 \pi)^{1 / 2}}\left[1+\left(\mathrm{e}^{-h}-1\right)\right. \\
& \left.\left.\times \int_{-\infty}^{K} \frac{\mathrm{d} \lambda}{[2 \pi(1-q)]^{1 / 2}} \exp \left(-\frac{(\lambda+t \sqrt{q})^{2}}{2(1-q)}\right)\right]^{n}\right\}
\end{aligned}
$$

and

$G_{1}(\mathrm{i} F, \mathrm{i} E)=n E+\frac{1}{2} n \ln (2 \pi)-\frac{1}{2} n \ln (2 E+F)-\frac{1}{2} \ln [1-F n /(2 E+F)]$.

In the limit, $n \rightarrow 0$, one gets,

$$
\begin{aligned}
G_{0}(q)=n \int_{-\infty}^{\infty} & \frac{\exp \left(-t^{2} / 2\right) \mathrm{d} t}{(2 \pi)^{1 / 2}} \\
& \times \ln \left(1+\left(\mathrm{e}^{-h}-1\right) \int_{-\infty}^{\left(K-1 q^{1 / 2}\right)(1-q)^{-1 / 2}} \exp \left(-\lambda^{2 / 2}\right)(2 \pi)^{-1 / 2} \mathrm{~d} \lambda\right)
\end{aligned}
$$

and

$$
G_{1}(q)=n\left[E+\frac{1}{2} \ln 2 \pi-\frac{1}{2} \ln (2 E+F)+\frac{1}{2} F /(2 E+F)\right] .
$$

This completes the calculation in the spherical case. In the Ising case, the calculation is almost the same. The differences are the following. The variables $\varepsilon_{\alpha}$ disappear everywhere (in (A1.2), (A1.5), (A1.6) and (A1.9)) so that $G_{1}$ in (A1.11) becomes a 
function of the $\varphi_{\alpha \beta}$ only. The integrals over the $J_{i j}^{\alpha}$ are replaced by sums over the two values +1 and -1 . Therefore, (A1.11) becomes

$$
\exp G_{1}\left(\left\{\varphi_{\alpha \beta}\right\}\right)=\sum_{J_{\alpha}= \pm 1} \exp \left(-\mathrm{i} \sum_{\alpha<\beta} \varphi_{\alpha \beta} J^{\alpha} J^{\beta}\right) \text {. }
$$

Assuming the replica symmetry,

$$
\varphi_{\alpha \beta}=\mathrm{i} F \quad \alpha \neq \beta
$$

one gets

$\exp G_{1}(F)=\exp \left(-\frac{1}{2} n F\right) \int \frac{\mathrm{d} u}{(2 \pi)^{1 / 2}} \exp \left\{-u^{2} / 2+n \ln [2 \cosh (u \sqrt{F})]\right\}$

Everything else remains the same and, in particular, $G_{0}$ is unchanged.

\section{Appendix 2}

In this appendix, the transverse eigenvectors and eigenvalues of the matrices, $\partial^{2} G_{0} / \partial q_{\alpha \beta} \partial q_{\gamma \delta}$ and $\partial^{2} G_{1} / \partial\left(\mathrm{i} \varphi_{\alpha \beta}\right) \partial\left(\mathrm{i} \varphi_{\gamma \delta}\right)$ will be calculated. From equation (A1.10)

$$
\frac{\partial^{2} G_{0}}{\partial q_{\alpha \beta} \partial q_{\gamma \delta}}=\left\langle x^{\alpha} x^{\beta} x^{\gamma} x^{\delta}\right\rangle-\left\langle x^{\alpha} x^{\beta}\right\rangle\left\langle x^{\gamma} x^{\delta}\right\rangle
$$

where \langle\rangle is defined

$$
\begin{aligned}
\langle f(x)\rangle \equiv \prod_{\alpha}\left(\mathrm{e}^{-h} \int_{-\infty}^{\kappa} \frac{\mathrm{d} \lambda^{\alpha}}{2 \pi}+\int_{K}^{\infty} \frac{\mathrm{d} \lambda^{\alpha}}{2 \pi}\right) \int_{-x}^{\infty} \mathrm{d} x^{\alpha} f(x) \\
\quad \times \exp \left(\mathrm{i} \lambda^{\alpha} x^{\alpha}-\frac{1}{2} \sum_{\alpha}\left(x^{\alpha}\right)^{2}-\sum_{\alpha<\beta} q^{\alpha \beta} x^{\alpha} x^{\beta}\right) \\
\times\left[\prod_{\alpha}\left(\mathrm{e}^{-h} \int_{-x}^{K} \frac{\mathrm{d} \lambda^{\alpha}}{2 \pi}+\int_{K}^{\infty} \frac{d \lambda^{\alpha}}{2 \pi}\right) \int_{-\infty}^{\infty} \mathrm{d} x^{\alpha}\right. \\
\left.\times \exp \left(i \lambda^{\alpha} x^{\alpha}-\frac{1}{2} \sum_{\alpha}\left(x^{\alpha}\right)^{2}-\sum_{\alpha<\beta} q^{\alpha \beta} x^{\alpha} x^{\beta}\right)\right]^{-1} .
\end{aligned}
$$

At the replica-symmetric saddle point, (A2.1) can take three possible values:

$$
\begin{array}{rlrl}
P=\frac{\partial^{2} G_{0}}{\partial q_{\alpha \beta} \partial q_{\alpha \beta}} & =\int \frac{\mathrm{d} t \exp \left(-t^{2} / 2\right)}{(2 \pi)^{1 / 2}}\left[\left(\overline{x^{2}}[t]\right)^{2}\right]-\left(\int \frac{\mathrm{d} t \exp \left(-t^{2} / 2\right)}{(2 \pi)^{1 / 2}}(\bar{x}[t])^{2}\right)^{2} \\
Q=\frac{\partial^{2} G_{0}}{\partial q_{\alpha \beta} \partial q_{\alpha \gamma}} & =\int \frac{\mathrm{d} t \exp \left(-t^{2} / 2\right)}{(2 \pi)^{1 / 2}}\left[\overline{x^{2}}(t)(\bar{x}[t])^{2}\right] \\
& -\left(\int \frac{\mathrm{d} t \exp \left(-t^{2} / 2\right)}{(2 \pi)^{1 / 2}}(\bar{x}[t])^{2}\right)^{2} & \beta \neq \gamma \\
R=\frac{\partial^{2} G_{0}}{\partial q_{\alpha \beta} \partial q_{\gamma \delta}} & =\int \frac{\mathrm{d} t \exp \left(-t^{2} / 2\right)}{(2 \pi)^{1 / 2}} \bar{x}^{4}[t] \\
& -\left(\int \frac{\mathrm{d} t \exp \left(-t^{2} / 2\right)}{(2 \pi)^{1 / 2}}(\bar{x}[t])^{2}\right)^{2} & \alpha \neq \gamma, \beta \neq \delta
\end{array}
$$


where $\overline{f(x)}[t]$ is defined

$$
\begin{aligned}
\overline{f(x)}[t]=\left(\mathrm{e}^{-h}\right. & \left.\int_{-x}^{x} \frac{\mathrm{d} \lambda}{2 \pi}+\left(1-\mathrm{e}^{-h}\right) \int_{K-q^{1 / 2},}^{x} \frac{\mathrm{d} \lambda}{2 \pi}\right) \int_{-x}^{\infty} \mathrm{d} x f(x) \exp \left[\mathrm{i} x \lambda-\frac{1}{2}(1-q) x^{2}\right] \\
& \times\left[\left(\mathrm{e}^{-h} \int_{-\infty}^{\infty} \frac{\mathrm{d} \lambda}{2 \pi}+\left(1-\mathrm{e}^{-h}\right) \int_{K-q^{1 / 2},}^{x} \frac{\mathrm{d} \lambda}{2 \pi}\right)\right. \\
& \left.\times \int_{-\infty}^{\infty} \mathrm{d} x \exp \left[\mathrm{i} x \lambda-\frac{1}{2}(1-q) x^{2}\right]\right]^{-1}
\end{aligned}
$$

since the denominator of equation (A2.2) tends to one in the limit $n \rightarrow 0$ and equation (A2.4) is obtained using the same method as in appendix 1. The matrix (A2.1) is thus of the same form as that in the de Almeida-Thouless calculation (1978) and the transverse eigenvectors $\eta^{\alpha \beta}$ have the form,

$$
\begin{array}{ll}
\eta^{\alpha_{0} \beta_{0}}=c & \\
\eta^{\alpha_{0} \alpha}=\eta^{\alpha \beta_{0}}=d & \alpha \neq \alpha_{0}, \beta_{0} \\
\eta^{\alpha \beta}=e & \alpha, \beta \neq \alpha_{0}, \beta_{0}
\end{array}
$$

where $\alpha_{0}$ and $\beta_{0}$ are a particular pair of replicas. In order that the eigenvector is normal to the $n$ eigenvectors which are degenerate with the longitudinal eigenvectors, the conditions

$$
c=(2-n) d \quad d=\frac{1}{2}(3-n) e
$$

must be satisfied. Substitution of conditions (A2.6) into the eigenvalue equation gives an $n(n-3) / 2$-fold degenerate eigenvalue

$$
P-2 Q+R=\int_{-\infty}^{\infty} \mathrm{d} t \exp \left(-t^{2} / 2\right)(2 \pi)^{-1 / 2}\left(\overline{x^{2}}(t)-(\bar{x}[t])^{2}\right)^{2} .
$$

The evaluation of this integral in the limit $q \rightarrow 1$ is similar to the method used in appendix 1. In this limit, the integral over $t$ is dominated by values of $t$,

$$
0<K-\sqrt{q} t<x
$$

and

$$
\begin{gathered}
\bar{x}[t]=\left(1-\mathrm{e}^{-h}\right) \mathrm{i} \exp \left[-(K-\sqrt{q} t)^{2} / 2(1-q)\right] \\
\times[2 \pi(1-q)]^{-1 / 2}\left(\mathrm{e}^{-h}+\left(1-\mathrm{e}^{-h}\right) \int_{\left(K-q^{1 / 2} t\right)(1-q)^{-1 / 2}}^{\infty} \mathrm{d} \lambda(2 \pi)^{-1 / 2} \mathrm{e}^{-\lambda^{2} / 2}\right)^{-1} \\
\overline{x^{2}}[t]=-\left(1-\mathrm{e}^{-h}\right) \exp \left[-(K-\sqrt{q} t)^{2} / 2(1-q)\right](K-\sqrt{q} t)\left[2 \pi(1-q)^{3}\right]^{-1 / 2} \\
\times\left(\mathrm{e}^{-h}+\left(1-\mathrm{e}^{-h}\right) \int_{\left(K-q^{1 / 2} t\right)(1-q)^{-1 / 2}}^{x} \mathrm{~d} \lambda(2 \pi)^{-1 / 2} \mathrm{e}^{-\lambda^{2} / 2}\right)^{-1}
\end{gathered}
$$

and so $\gamma_{1}$ diverges as $q \rightarrow 1$,

$$
\gamma_{1}\left(\alpha_{\mathrm{c}}\right)=\frac{1}{(1-q)^{2}} \int_{K-x}^{\kappa} \mathrm{d} t \exp \left(-t^{2} / 2\right)(2 \pi)^{-1 / 2} .
$$

The calculation of the transverse eigenvalues of $\partial^{2} G_{1} / \partial \mathrm{i} \varphi_{\alpha \beta} \partial \mathrm{i} \varphi_{\gamma \delta}$ is similar. The eigenvectors are again given by $(\mathrm{A} 2.5)$ and $(\mathrm{A} 2.6)$ and the eigenvalue by $P^{\prime}-2 Q^{\prime}+R^{\prime}$ 
where

$$
\begin{array}{ll}
P^{\prime}=\frac{\partial^{2} G_{1}}{\partial\left(\mathrm{i} \varphi_{\alpha \beta}\right) \partial\left(\mathrm{i} \varphi_{\alpha \beta}\right)} & \\
Q^{\prime}=\frac{\partial^{2} G_{1}}{\partial\left(\mathrm{i} \varphi_{\alpha \beta}\right) \partial\left(\mathrm{i} \varphi_{\alpha \gamma}\right)} & \beta \neq \gamma \\
R^{\prime} & =\frac{\partial^{2} G_{1}}{\partial\left(\mathrm{i} \varphi_{\alpha \beta}\right) \partial\left(\mathrm{i} \varphi_{\gamma \delta}\right)}
\end{array}
$$

and $\gamma_{2}$ can be found easily

$$
\gamma_{2}=(1-q)^{2}
$$

\section{References}

de Almeida J R and Thouless D J 1978 J. Phys. A: Math. Gen. 11983

Amit D J, Gutfreund H and Sompolinsky H 1985a Phys. Rev. Lett. 551530

- 1985b Phys. Rev. A 321007 1987a Ann. Phys., NY 17330 1987b Phys. Rev. A in press

Bray A J and Moore M A 1980 J. Phys. C: Solid State Phys. 13 L469

1981 J. Phys. C: Solid State Phys. 141313

Cover T M 1965 IEEE Trans. Electron. Comput. EC-14 326

De Dominicis C, Gabay M, Garel T and Orland H 1980 J. Physique 41933

Derrida B and Gardner E 1986 J. Physique 47959

Diederich S and Opper M 1987 Phys. Rev. Lett. 58949

Edwards S F and Anderson. P W 1975 J. Phys. F: Met. Phys. 5965

Ettelaie R and Moore M A 1985 J. Physique Lett. 461893

Gardner E 1986 J. Phys. A: Math. Gen. 19 L1047

- 1987 Europhys. Lett. 4481

_- 1988a J. Phys. A: Math. Gen. 21257 1988 b Preprint Edinburgh, in preparation

Gardner E, Stroud N and Wallace D J 1987 Preprint Edinburgh 87/394

Hebb D O 1949 The Organisation of Behaviour (New York: Wiley)

Hopfield J J 1982 Proc. Natl Acad. Sci. USA 792554

Kanter I and Sompolinsky H 1987 Phys. Rev. A 35380

Kohonen T 1984 Self Organisation and Associative Memory (Berlin: Springer)

Krauth W and Mézard M 1987 J. Phys. A: Math. Gen. 20 L745

Little W A 1964 Math. Biosci. 19101

Mézard M, Nadal J P and Toulouse G 1986 J. Physique 471457

Minsky M and Papert S 1969 Perceptrons (Cambridge, MA: MIT Press)

Parisi G 1986 J. Phys. A: Math. Gen. 19 L617

Personnaz L, Guyon I and Dreyfus G 1985 J. Physique Lett. 16 L359

Poppel G and Krey U 1987 Preprint Regensburg

Rosenblatt F 1962 Principles of Neurodynamics (New York: Spartan Books)

Sherrington D and Kirkpatrick S 1975 Phys. Rev. Lett. 321792

Toulouse G, Dehaene S and Changeux J P 1986 Proc. Natl Acad. Sci. USA 831695

Venkatesh S 1986a Proc. Conf. on Neural Networks for Computing, Snowbird, UT (AIP Conf. Proc. 151) ed J S Denker (New York: AIP)

1986b PhD thesis California Institute of Technology 